\title{
SCF/C-Kit/JNK/AP-1 Signaling Pathway Promotes Claudin-3 Expression in Colonic Epithelium and Colorectal Carcinoma
}

\author{
Yaxi Wang ${ }^{1,2}$, Tingyi Sun ${ }^{1,2,3}$, Haimei Sun ${ }^{1,2,3}$, Shu Yang ${ }^{1,2,3}$, Dandan $\mathrm{Li}^{1,2}$ and \\ Deshan Zhou 1,2,3,* \\ 1 Department of Histology and Embryology, School of Basic Medical Sciences, Capital Medical University, \\ Beijing 100069, China; wangyaxi1@sina.com (Y.W.); styj211@ccmu.edu.cn (T.S.); haimei@ccmu.edu.cn (H.S.); \\ sheilayslamb@aliyun.com (S.Y.); lidan0135@sina.com (D.L.) \\ 2 Beijing Key Laboratory of Cancer Invasion and Metastasis Research, Beijing 100069, China \\ 3 Cancer Institute of Capital Medical University, Beijing 100069, China \\ * Correspondence: zhouds08@ccmu.edu.cn; Tel.: +86-10-8395-0079
}

Academic Editor: Atsushi Matsuzawa

Received: 4 March 2017; Accepted: 30 March 2017; Published: 6 April 2017

\begin{abstract}
Claudin-3 is a major protein of tight junctions (TJs) in the intestinal epithelium and is critical for maintaining cell-cell adhesion, barrier function, and epithelium polarity. Recent studies have shown high claudin-3 levels in several solid tumors, but the regulation mechanism of claudin-3 expression remains poorly understood. In the present study, colorectal cancer (CRC) tissues, HT-29 and DLD-1 CRC cell lines, CRC murine model (C57BL/ 6 mice) and c-kit loss-of-function mutant mice were used. We demonstrated that elevated claudin-3 levels were positively correlated with highly expressed c-kit in CRC tissues based upon analysis of protein expression. In vitro, claudin-3 expression was clearly increased in CRC cells by overexpressed c-kit or stimulated by exogenous recombinant human stem cell factor (rhSCF), while significantly decreased by the treatment with c-kit or c-Jun N-terminal kinase (JNK) inhibitors. Chromatin immunoprecipitation (ChIP) and luciferase reporter assay showed that SCF/c-kit signaling significantly promoted activator protein-1 (AP-1) binding with $C L D N-3$ promoter and enhanced its transcription activity. Furthermore, decreased expression of claudin-3 was obtained in the colonic epithelium from the $c$-Kit loss-of-function mutant mice. In conclusion, SCF/c-kit-JNK/AP-1 signaling pathway significantly promoted claudin-3 expression in colonic epithelium and CRC, which could contribute to epithelial barrier function maintenance and to CRC development.
\end{abstract}

Keywords: claudins; colorectal cancer; c-kit; claudin-3; c-Jun N-terminal kinase; activator protein-1

\section{Introduction}

Claudins are the major components of tight junctions (TJs) in the epithelium, and 27 family members widely distribute in different tissues. It is well known that claudins play important roles in maintaining cell-cell adhesion, barrier function, and epithelial physiological polarity [1,2]. Disruption of claudins frequently leads to anomalous structure and dysfunction of TJs, which may cause barrier related diseases [3]. Among the claudin members, claudin-3 is mainly expressed in basolateral membrane and the apical of the epithelium and is a main protein of TJs in the intestinal epithelium. Its absence often results in leaky paracellular permeability, which plays an important role in the pathogenesis of inflammatory bowel diseases [4]. In recent years, it has been reported that claudin-3 was highly expressed in ovarian cancers, but hardly detected in normal ovarian epithelium [5], thereby, claudin-3 was considered to be a promising target for diagnosis and treatment in ovarian cancers [6]. 
Furthermore, claudin-3 was also overexpressed in breast [7] and prostate [8] cancers. In fact, claudin members are often highly expressed in a variety of gastrointestinal tumors, for example, claudin-3, -4 , and -7 are highly expressed in early esophageal adenocarcinoma [9], claudin- 1 is highly expressed in gastric cancer [10], and claudin-1 and -2 are highly expressed in colorectal cancer (CRC) [11]. These results indicate that abnormally elevated claudins are positively correlated with tumorigenesis and progression of gastrointestinal tumors [12]. However, the molecular mechanism underlying the regulation of claudins expression remains poorly understood.

Recent studies have suggested that the activation of signaling molecules belonging to the receptor tyrosine kinase (RTK) superfamily are involved in up-regulating claudins expression during inflammation and several cancers. For instance, epidermal growth factor (EGF)/epidermal growth factor receptor (EGFR) has been reported to be responsible for elevated claudin-2 expression in CRC [13] and lung cancer [14]. It was further found that the activation of mitogen-activated protein kinase (MAPK), the downstream hub molecules of RTKs, was common in the abnormal expression of claudins [15-17]. It is worth mentioning that aberrant activation of c-kit, another RTK superfamily member and its ligand, stem cell factor (SCF), signaling was considered to play key roles in tumor cell proliferation, migration and invasion, which contributed to the aggressive biological behaviors of CRC and the shortened survival period of CRC patients [18]. Noticeably, claudin-3 was also overexpressed in these patients [19], implying a possibility that SCF/c-kit signaling might be a potential factor in the regulation of claudin-3 expression in colonic epithelium and CRC.

Therefore, the main aim of this study is to investigate the detailed mechanism of claudin-3 expression regulated by SCF/c-kit signaling. Our results indicated that high expression of claudin-3 was positively correlated with the activation of SCF/c-kit signaling. Furthermore, activation of c-Jun N-terminal kinase (JNK) and activator protein-1 (AP-1) molecules were found to be able to effectively promote claudin-3 expression in CRC cells. These results provide insight into the molecular mechanisms underlying the regulation of claudin-3 expression in the intestinal epithelium and in several tumor tissues.

\section{Results}

\subsection{Clauin-3 Level Is Positively Correlated with C-Kit Expression in Colorectal Cancer (CRC) Tissues}

Previous studies suggested that activation of c-kit signaling [20] and overexpression of claudin-3 [21] were both involved in CRC development. To clarify the correlation between c-kit and claudin-3, we collected 12 tumor samples from CRC patients and matched adjacent normal tissues. Claudin-3 mRNA and protein levels in CRC tissues were significantly elevated in 8 out of 12 patients. Simultaneously, c-kit protein was also shown to be up-regulated in these eight CRC samples (Figure 1A). The same phenomenon was also seen in mice CRC, and there was a significant positive correlation between c-kit and claudin-3 protein expressions $(r=0.6, p<0.01$; Figure 1B). Furthermore, immunofluorescence staining showed a high expression of claudin- 3 in mouse CRC tissues compared to the normal tissues (Figure 1C). 


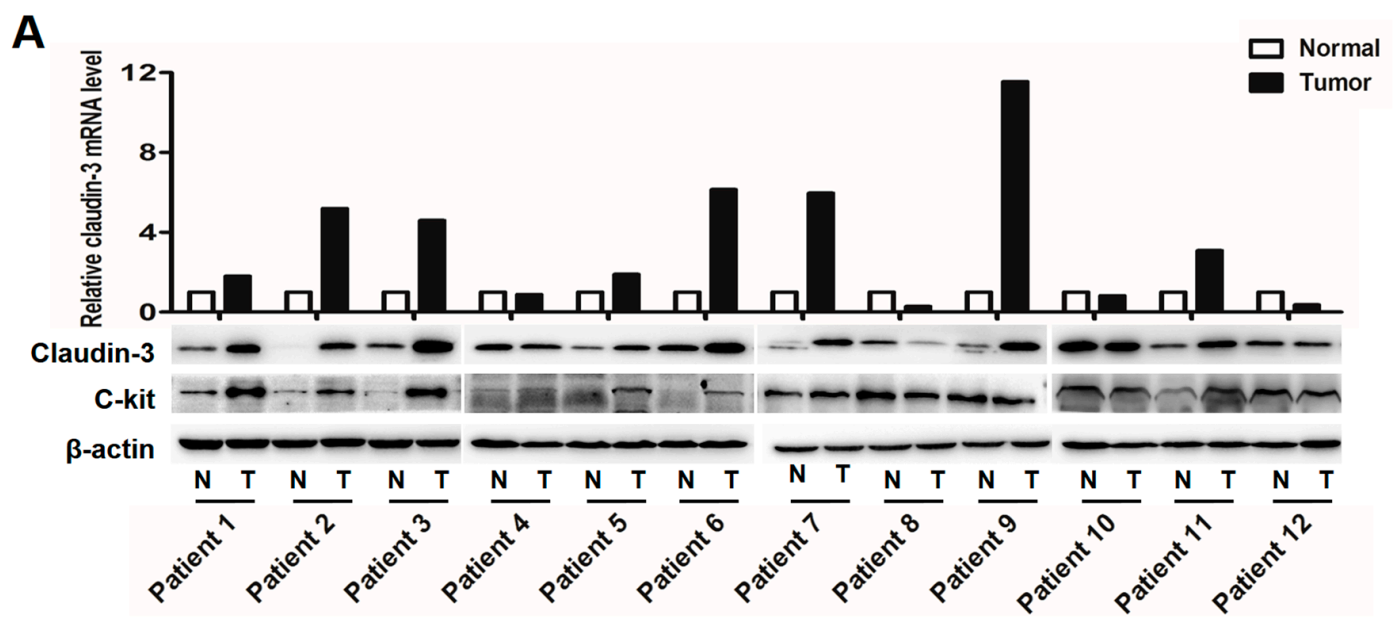

B
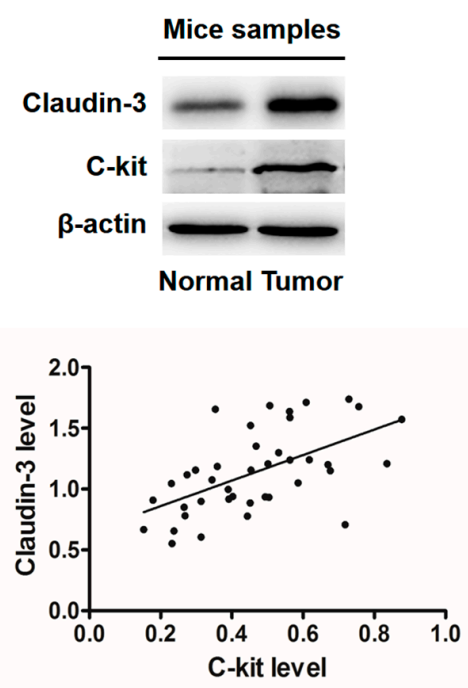

C

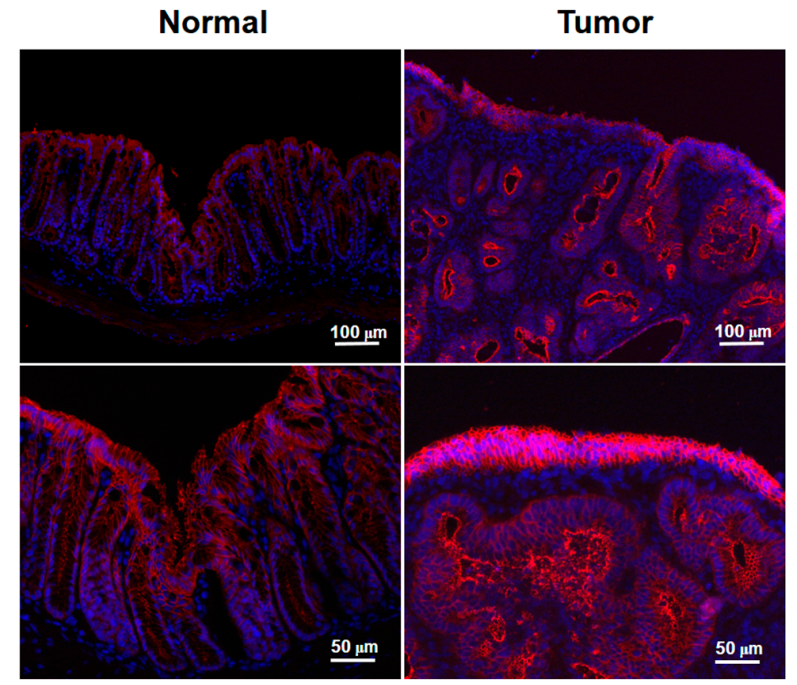

Claudin-3; DAPI

Figure 1. Claudin-3 and c-kit expressions are positively correlated in colorectal cancer (CRC) tissues. (A) Real-time polymerase chain reaction (PCR) was performed to detect CLDN-3 mRNA level in CRC tissues and adjacent normal tissues from 12 patients. Glyceraldehyde-3-phosphate dehydrogenase (GAPDH) was used as the internal control. Normalized CLDN-3 mRNA expression was shown in the column graph (N, normal; $\mathrm{T}$, tumor). Western blot showing the protein expression of claudin-3 which was consistent with its mRNA level; (B) Western blot revealed that c-kit and claudin-3 were both highly expressed in CRC tissues from mice. $\beta$-Actin was used as the loading control. A significant positive correlation between c-kit and claudin-3 protein expressions was observed by Spearman correlation analysis in mouse CRC tissues $(n=20, r=0.6, p<0.01)$; (C) Immunofluorescence staining showed elevated claudin-3 expression in mouse CRC tissues.

\subsection{Stem Cell Factor (SCF)/C-Kit Signaling Significantly Increases Claudin-3 Expression}

To clarify the role of SCF/c-kit signaling, claudin-3 expression was examined in HT-29 cells which highly expressed c-kit. As expected, significantly increased expressions of claudin-3 protein were observed in a dose- and time-dependent manner after rhSCF administration (Figure 2A,B). Likewise, overexpression of c-kit via lentivirus mediation in HT-29 cells significantly increased claudin-3 mRNA and protein level (Figure 2C). While Imatinib, a specific tyrosine kinase inhibitor, clearly down-regulated the basal and rhSCF-induced high expression of claudin-3. The protein expression was consistent with their mRNA level (Figure 2D). 
A

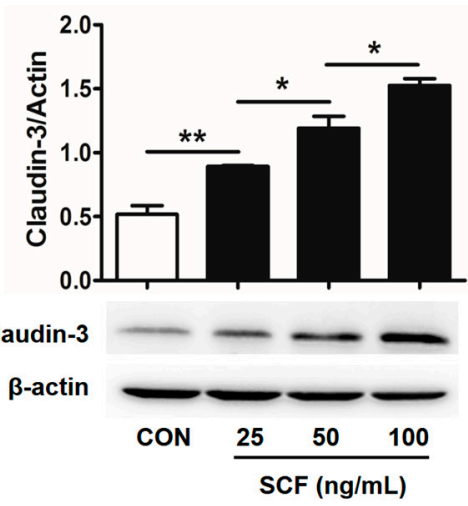

C

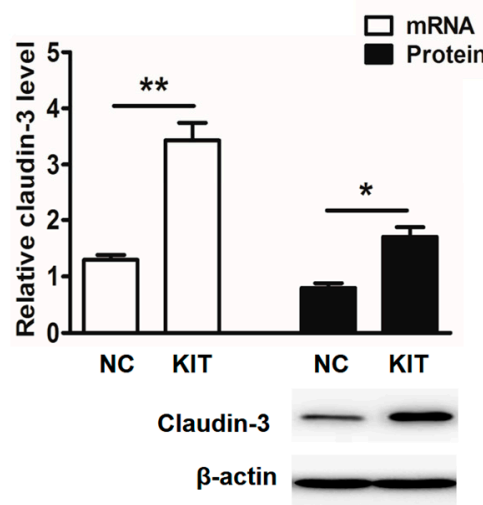

B

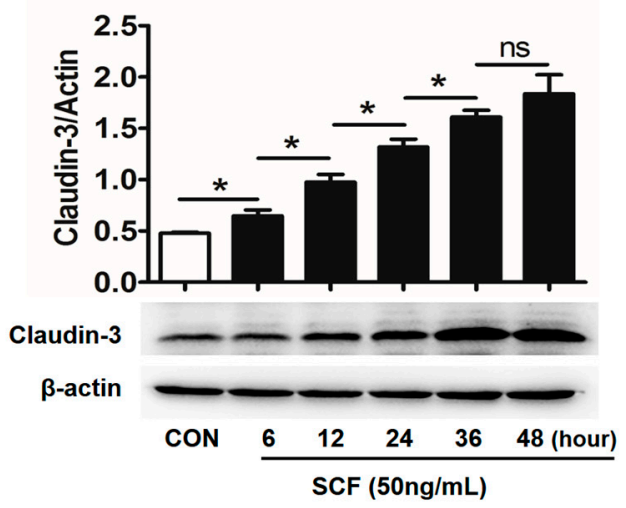

D

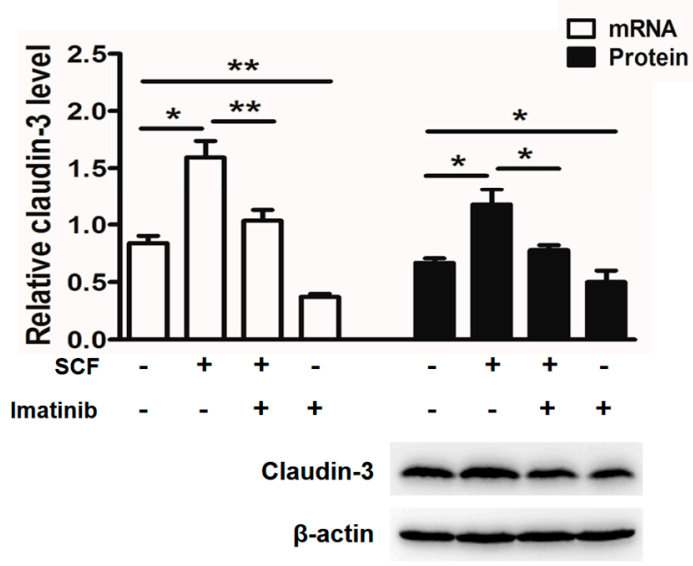

Figure 2. Stem cell factor (SCF)/c-kit signaling clearly increases claudin-3 expression in HT-29 cells. Activated SCF/c-kit signaling by exogenous rhSCF in HT-29 cells increased claudin-3 expression in a dose- (A) and time- (B) dependent manner $\left({ }^{*} p<0.05,{ }^{* *} p<0.01\right.$, ns $p>0.05$, Con, control; SCF, stem cell factor); (C) Lentivirus-mediated overexpression of c-kit clearly promoted claudin-3 mRNA and protein expressions (NC, lentivirus-control; KIT, lentivirus-c-kit); (D) HT-29 cells were incubated in the absence and presence of rhSCF (50 ng/mL), Imatinib $(2 \mu \mathrm{M})$, or rhSCF plus Imatinib for $36 \mathrm{~h}$. It'was clearly seen that Imatinib treatment reduced the basal and rhSCF-induced claudin-3 mRNA and protein expressions. All the values are mean \pm SEM of three independent experiments ${ }^{*} p<0.05$, ** $p<0.01$, ns $p>0.05$, Con, control).

In view of the involvement of MAPK pathways in SCF/c-kit signaling, HT-29 cells were treated with specific inhibitor of each of the MAPK molecules. The results showed that although rhSCF administration activated all three MAPK molecules (Supplementary Figure S1), blockage of JNK, rather than p38 or extracellular signal-regulated kinases (ERK), significantly attenuated the claudin-3 expression in the basal and rhSCF-treated case (Figure 3A). Similar results were also obtained from DLD-1 cells (Figure 3B and Supplementary Figure S2). Collectively, our results suggested that SCF/c-kit-MAPK/JNK signaling pathway could promote claudin-3 expression in CRC cells. 
A

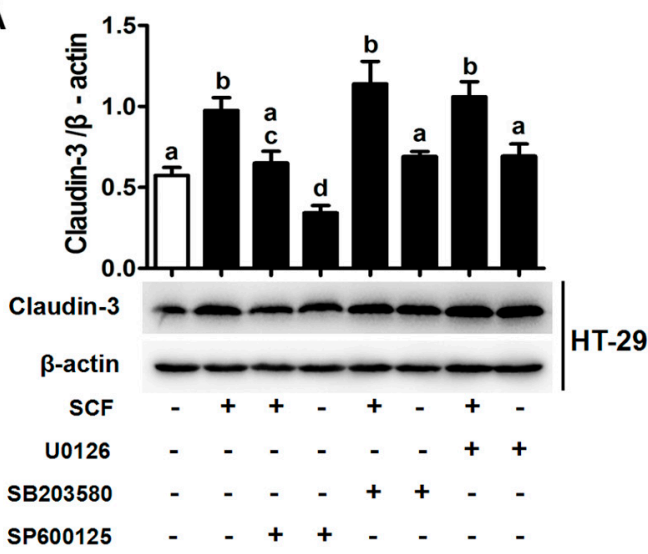

B

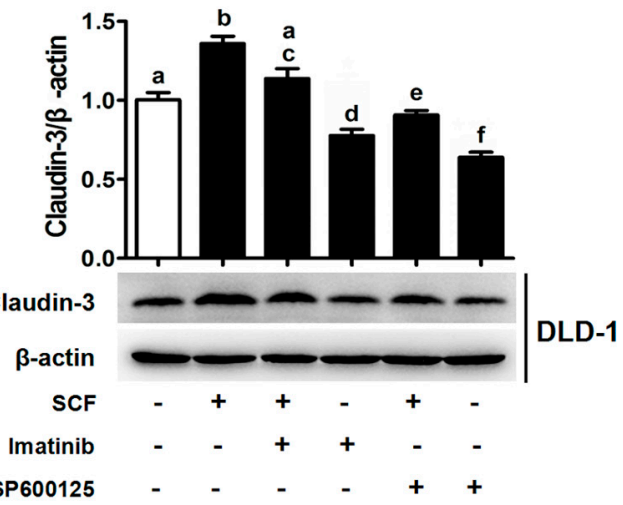

Figure 3. SCF/c-kit signaling increases claudin-3 expression via the c-Jun N-terminal kinase (JNK) pathway. (A) HT-29 cells were exposed to rhSCF alone or in combination with U0126 (ERK inhibitor), SB203580 (p38 inhibitor), or SP600125 (JNK inhibitor), respectively. Only SP600125 treatment significantly abrogated the rhSCF-induced claudin-3 expression; (B) The same experiments were performed in DLD-1 cells. Claudin-3 expression was increased after rhSCF administration, while decreased by the treatment with c-kit or JNK inhibitors. All the values are mean $\pm \mathrm{SEM}$ of three independent experiments ( $n=3$, different letters indicate significant differences between groups).

\subsection{Activator Protein-1 (AP-1) Is Activated by c-Jun N-Terminal Kinase (JNK) in CRC Tissues}

Next, we searched the key transcriptional factors that could regulate CLDN-3 transcription by using the on-line transcription factor prediction databases (JASPAR, http://jaspar.genereg.net/). We found that $C L D N-3$ promoter region harbored the binding sites of AP-1/c-Jun, which was an important downstream transcription factor of JNK signaling. The transcriptional complex AP-1 consists of two subunits, c-Fos and c-Jun. Its activation predominantly depends on its dimer composition, Jun-Jun homodimers or Jun-Fos heterodimers. Thus, as the major component of AP-1, c-Jun phosphorylation plays an important role in AP-1 activation and is usually used as a marker of activated AP-1 [22]. We found that phosphorylation of JNK and c-Jun were both increased in CRC tissues which highly expressed c-kit and claudin-3 (Figure 4A,B). C-Jun phosphorylation was increased by rhSCF administration while decreased after pretreated with SP600125 in HT-29 cells and DLD-1 cells, consistent with the alterations of claudin-3 expression (Supplementary Figures S1 and S2). Furthermore, HT-29 cells treated with phorbol 12-myristate 13-acetate (TPA), a stimulator of AP-1, clearly elevated claudin-3 expression (Figure 4C). 
A

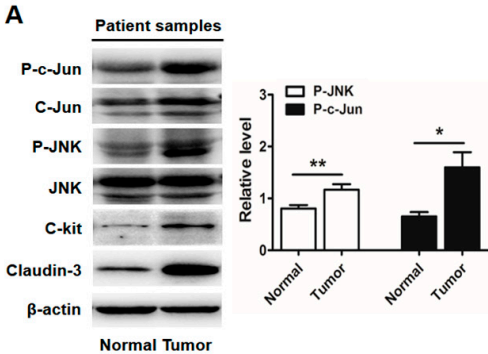

B

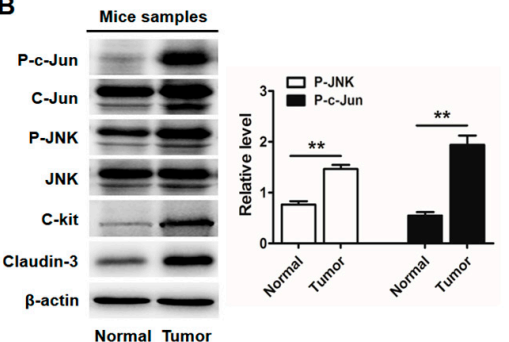

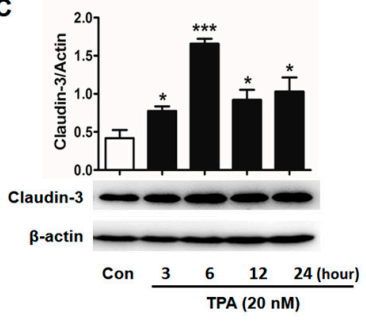

Figure 4. C-Jun mediates the c-kit/JNK-dependent increase in claudin-3 expression. Western blot indicated that p-JNK and p-c-Jun were increased in colorectal cancer (CRC) tissues of patients (A) and mice (B) in which both c-kit and claudin-3 were highly expressed. The levels of p-JNK and p-c-Jun were respectively represented relative to the value of JNK and c-Jun; (C) HT-29 cells were treated with phorbol 12-myristate 13-acetate (TPA) $(20 \mathrm{nM})$ for different time periods and claudin-3 level was significantly elevated after TPA administration (TPA 3, 6, 12, $24 \mathrm{~h}$ vs. Con.). All the values are mean \pm SEM of three independent experiments ( ${ }^{*} p<0.05,{ }^{* *} p<0.01, * * * p<0.001$, Con., control).

\subsection{AP-1/c-Jun Enhances CLDN-3 Promoter Activity}

Three putative AP-1/c-Jun binding sites in the promoter region of the human CLDN-3 gene were predicted (Figure 5A). To further identify the role of AP-1/c-Jun activation on claudin-3 expression, pGL3-Cldn3 luciferase reporter gene construct with c-Jun plasmid were co-transfected into HEK293T cells and the results are shown in Figure 5B. Over-expressed c-Jun obviously enhanced the luciferase activity of $C L D N-3$ promoter, which was abrogated when mutating each c-Jun binding site. These results indicate that AP-1/c-Jun could increase CLDN-3 transcription activity and that each of the three c-Jun binding sites was of great significance.
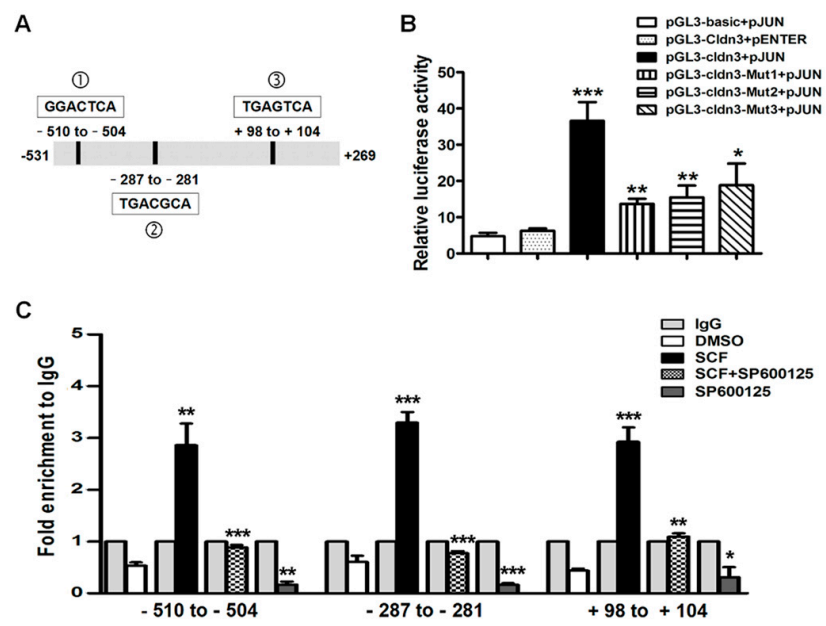

Figure 5. Binding ability of c-Jun to CLDN-3 promoter is mediated by c-kit/JNK signaling pathway. (A) Three c-Jun binding sites were predicted in the CLDN-3 promoter flanking region; (B) The dual luciferase reporter assays were performed at $24 \mathrm{~h}$ after transfection. C-Jun upregulated luciferase activity of CLDN-3 promoter in $293 \mathrm{~T}$ cells, but this role was abolished by mutating each binding site (pGL3-basic + pJun/pGL3-Cldn3 + pEnter vs. pGL3-Cldn3 + pJun, pGL3-Cldn3 + pJun vs. pGL3-Cldn3-Mut 1/2/3 + pJun); (C) HT-29 cells were treated with DMSO, rhSCF, SP600125, and rhSCF plus SP600125, respectively, followed by chromatin immunoprecipitation (ChIP) assays. Binding of c-Jun with the CLDN-3 promoter was effectively enhanced by activation of the SCF/c-kit/JNK signaling pathway. The values were shown relative to immunoglobulin $\mathrm{G}(\mathrm{IgG})$ which was set to 1 (SCF/SP600125 vs. DMSO, SCF + SP600125 vs. SCF). All the values are mean \pm SEM of three independent experiments $\left({ }^{*} p<0.05,{ }^{* *} p<0.01,{ }^{* * *} p<0.001\right)$. 


\subsection{SCF/C-Kit/JNK Signaling Promotes the Binding of AP-1/c-Jun with CLDN-3 Promoter}

Chromatin immunoprecipitation (ChIP) assays were performed to determine whether AP-1/c-Jun could bind the promoter region of $C L D N-3$ with the anti-c-Jun antibody. Real-time PCR using three pairs of special primers against $C L D N-3$ promoter showed that rhSCF induced a 5.5 7.3 fold enrichment of AP-1/c-Jun at each binding region compared with the control group. In contrast, markedly diminished occupancy was observed when the cell was treated with JNK inhibitor, SP600125 (Figure 5C). Clearly, AP-1/c-Jun could directly bind to the CLDN-3 promoter, which was also influenced by c-kit/SCF/JNK signaling.

\subsection{Claudin-3 Decreases in Colonic Mucosa of c-Kit Loss-of-Function Mutant Mice}

In the present study, c-Kit loss-of-function mutant mice (Wads ${ }^{-/}$) were used for further examination of claudin-3 expression in vivo. Claudin-3 decreased in colonic mucosa, while other tight junction proteins, including claudin-1, $-2,-7$, occludin, and adhesion protein, $E$-cadherin, remained unchanged compared to the wild-type (WT) mice (Figure 6A). Similar results were obtained by immunofluorescence staining showing lower claudin-3 expression in colonic mucosa of the $\mathrm{Wads}^{-1-}$ mice (Figure 6B). These results further supported that SCF/c-kit signaling was crucial to maintain claudin-3 expression in the intestinal epithelium.
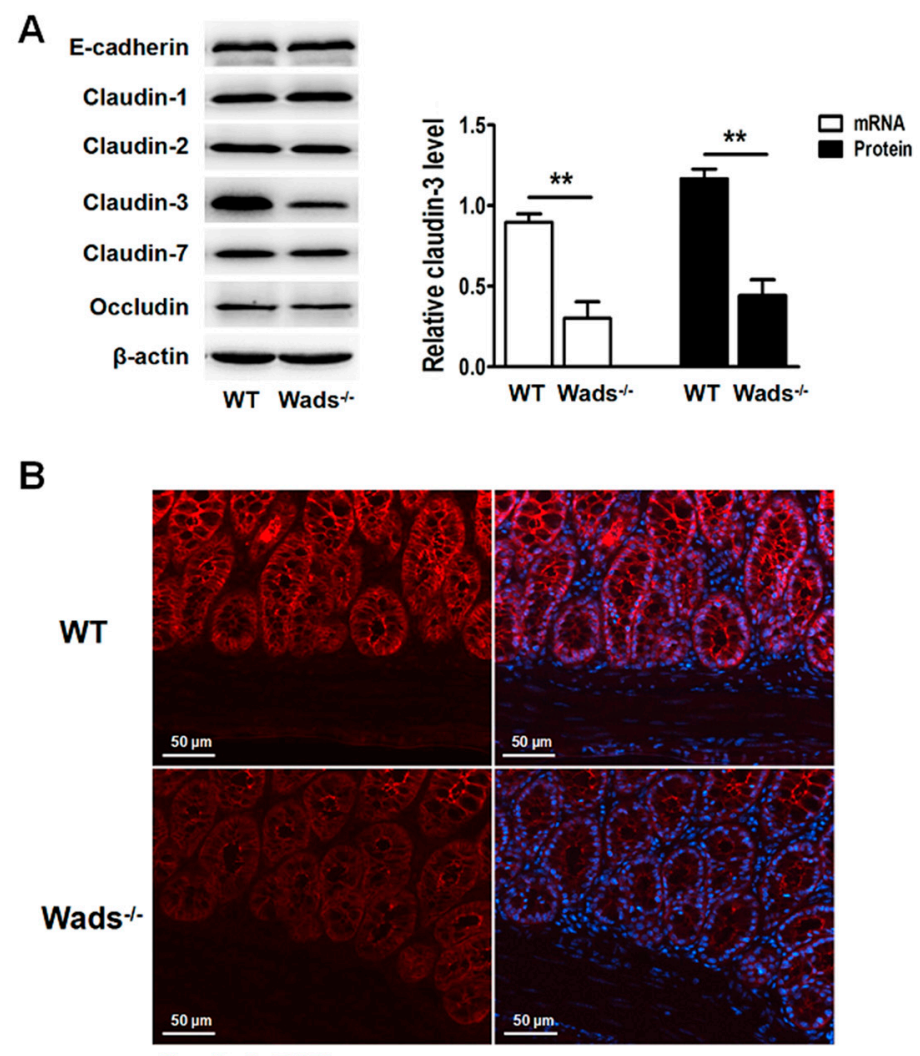

Claudin-3; DAPI

Figure 6. Claudin-3 is decreased in colonic epithelium of $c$-Kit loss-of-function mutant mice (Wads ${ }^{-/}$). (A) Expressions of $E$-cadherin, claudin-1, -2, -3, -7, and occludin in the colonic epithelium of Wads ${ }^{-/-}$ mice and wild-type (WT) mice examined by western blot. Only claudin-3 significantly decreased in $\mathrm{Wads}^{-/-}$mice compared to the WT mice. All the values are mean $\pm \mathrm{SEM}$ of three independent experiments $(n=5, * * p<0.01)$; (B) Claudin-3 expression was decreased in the colonic epithelium of $\mathrm{Wads}^{-/-}$mice compared to the WT mice, as determined by immunofluorescence staining. 


\section{Discussion}

TJs are polarized epithelial structures that are essential for keeping the epithelium more "tight" in organisms. Its breakdown leads to barrier dysfunction related diseases such as irritable bowel disease [4] and celiac disease [23]. Claudin-3 is an important protein of TJs and its absence impairs the blood-testis barrier during leptotene translocation [24]. Recent studies indicated potential functions of claudin-3 on tumorigenesis and revealed that high claudin-3 expression was correlated with shorter overall survival in several cancers [25]. In the present study, we observed that claudin-3 was obviously upregulated in the CRC tissues of patients and mouse models, which were consistent with other reports [26] in which high expression of claudin-3 was noted in CRC. However, information about the regulation of claudin-3 in the intestinal epithelium and tumor tissues is really limited. Previous studies suggested that highly-expressed claudin- 3 could be induced by epigenetic processes including DNA methylation and histone $\mathrm{H} 3$ acetylation [27].

In our study, we found that highly expressed claudin-3 was often associated with the activation of $\mathrm{SCF} / \mathrm{c}-\mathrm{kit}$ signaling, suggesting that this signaling might play an important role in the regulation of claudin-3 expression in CRC. This hypothesis was further confirmed by using c-Kit loss-of-function mutant mice that had weaker claudin-3 expression in the colonic mucosa compared to the WT mice. Our results were consistent with the earlier reports that RTK signaling participated in regulating claudins expression. For example, EGF/EGFR widely modulated claudins expression in various organs including lung [14], kidney [28], and colon [13]; insulin-like growth factor 1 (IGF-1)/insulin-like growth factor 1 receptor (IGF-1R) was responsible for the change of claudin-1 expression during bone differentiation [29]; vascular endothelial growth factor (VEGF)/vascular endothelial growth factor receptor (VEGFR) was identified as an upstream regulator of claudin-5 expression [30]. Thus, RTK signaling clearly contributes to the regulation of claudins expression in multiple cells and tissues.

Regarding the molecular mechanism of claudins under the regulation by RTK signaling, it was considered that their common downstream molecules, MAPK, including three subfamilies, ERK1/2, p38, and JNK1/2/3, played a critical role. Ikari et al. [14] reported that PI-3 kinase and ERK1/2 pathways mediated claudin-2 expression in lung cancers. Whereas, in the current study, we identified that SCF/c-kit signaling increased claudin-3 expression only by activating the JNK pathway in colonic epithelium and CRC cells. This difference may be due to the different cells and tissues. It is well known that JNK often induces c-Jun phosphorylation, a subfraction of AP-1, at N-terminal Ser63 and Ser73, leading to the formation of activated AP-1 dimers. We found that the increased phosphorylation of JNK and c-Jun was often accompanied by highly expressed claudin-3 in colonic epithelium and CRC cells. It was well known that AP-1, as a multifunctional transcription factor, frequently regulates the expression of multiple genes related to cell proliferation, differentiation, and apoptosis [31]. Accordingly, we predicted three putative AP-1 binding sites in the promoter region of human CLDN-3 gene and demonstrated that AP- 1 could bind to the $C L D N-3$ promoter region and that each of the three AP-1 binding sites significantly increase $C L D N-3$ transcription activity. Moreover, the HT-29 cells treated with rhSCF, TPA, or corresponding inhibitors also elevated or reduced claudin-3 levels, further confirming the role of AP-1 in claudin-3 expression. Taken together, we considered that SCF/c-kit-JNK-AP-1 signaling axis played a critical role in the regulation of claudin-3 expression in colonic epithelium and $\mathrm{CRC}$, which might contribute to maintaining epithelium barrier functions and body homeostasis, physiologically. However, the aberrant activation of these signal molecules would alter the claudin expression and function that are involved in the development of several solid tumors. It was reported that claudin-2 could form a complex with zonula occludens (ZO-1), ZO-1-associated protein (ZONAB), and cyclin D1 leading to enhancement of cell proliferation, which contributed to the development of lung cancer [32]. It enlightened us of the possibility that highly-expressed claudin-3 regulated by SCF/c-kit signaling might be involved in colorectal tumorigenesis. In addition, the interaction between the claudins with actin could alter cell movement [33], which might also be associated with tumor invasion and migration. Therefore, the detailed role and molecular mechanism of claudin-3 in CRC needs to be further explored. 


\section{Materials and Methods}

\subsection{Ethics Statement}

All animals' studies were carried out strictly under protocols approved by the Animal Care and Use Committee of Capital Medical University (Permit Number AEEI-2014-058, 17 June 2014). Every effort was made to minimize the number of animals used as well as their suffering.

\subsection{Patient Samples}

The clinical CRC samples, including 12 pairs of primary CRC tumors and their adjacent non-tumorous tissues, were collected immediately after surgical resection prior to any other therapeutic intervention at the Xuanwu Hospital Capital Medical University (Beijing, China). The study protocol was approved by the Clinical Research Ethics Committee of the Xuanwu Hospital Capital Medical University (Permit Number 2013-X-036, 20 July 2013). All patients were chemotherapy and radiation therapy naive and their samples were confirmed by pathological examination. The tissue samples were stored at $-80^{\circ} \mathrm{C}$ immediately after collection.

\subsection{Establishment of CRC Murine Model}

CRC models of C57BL/6 mice were established through azoxymethane (AOM, $10 \mathrm{mg} / \mathrm{kg}$ ) (Sigma-Aldrich, St. Louis, MO, USA) injection and followed by three periods of $2.5 \%$ dextran sodium sulfate (DSS; MP Biomedicals, Solon, OH, USA) in drink water, as previously described [20]. The mice were sacrificed at 12, 20,28, and 36 weeks after AOM injection, respectively. Half of the neoplasms were frozen in liquid nitrogen for protein and mRNA detections and the rest was fixed immediately in $4 \%$ paraformaldehyde for paraffin embedding or optimal cutting temperature (OCT) compound embedding. Sections were cut for immunofluorescence and/or hematoxylin-eosin (HE) staining.

\subsection{RNA Exraction and Real-Time PCR}

Total RNA was extracted from CRC tissues and cultured cells with TRIzol reagent (Life Technologies, Carlsbad, CA, USA). Reverse transcription reactions were performed using High-Capacity cDNA Reverse Transcription Kit (Life Technologies). Twenty microliter reactions were incubated in a Veriti 96-well Thermal Cycler (Life Technologies) for $40 \mathrm{~min}$ at $42{ }^{\circ} \mathrm{C}$ and $5 \mathrm{~min}$ at $85^{\circ} \mathrm{C}$. Real-time PCR was performed in an ABI 7500 real-time PCR system (Life Technologies) using Ultra SYBR Mixture with ROX (CWBiotech, Beijing, China). The following primers are listed in Supplementary Table S1. Twenty-five microliter reactions were incubated at $95{ }^{\circ} \mathrm{C}$ for $10 \mathrm{~min}$, followed by 40 cycles at $95^{\circ} \mathrm{C}$ for $10 \mathrm{~s}, 60^{\circ} \mathrm{C}$ for $10 \mathrm{~s}$, and $72{ }^{\circ} \mathrm{C}$ for $40 \mathrm{~s}$. All PCR reactions were run in triplicate. Relative gene expression was determined using comparative CT (2-DeltaDeltaCt calculation, $2^{-\Delta \Delta C \mathrm{t}}$ ) method.

\subsection{Western Blot}

Total protein was extracted from CRC tissues and cultured cells and subjected to immunoblotting, as previously described [20]. Primary antibodies including rabbit polyclonal anti-claudin-1 (1:1000), anti-claudin-2 (1:500), anti-claudin-3 (1:1000), and anti-claudin-7 (1:1000) were obtained from Abcam (Cambridge, MA, USA). Primary antibody rabbit polyclonal anti-occludin (1:400) was purchased from Invitrogen (Carlsbad, CA, USA). Primary antibodies including rabbit polyclonal anti-E-cadherin (1:1000), anti-c-kit (1:1000), anti-c-Jun (1:1000), anti-p-c-Jun (1:1000), anti-JNK (1:1000), and anti-p-JNK (1:1000) were obtained from Cell Signaling Technology (Beverly, MA, USA). Mouse monoclonal anti- $\beta$-actin (1:2000) was purchased from Santa Cruz Biotechnology (Santa Cruz, CA, USA). The proteins were detected using enhanced chemiluminescence (ECL) (ThermoFisher Scientific, Waltham, MA, USA) and viewed in Fusion FX Vilber Lourmat (Paris, France). 


\subsection{Immunofluorescence Staining}

Cryosections $(5 \mu \mathrm{m})$ were fixed with $4 \%$ paraformaldehyde for $20 \mathrm{~min}$ at $25^{\circ} \mathrm{C}$. Non-specific binding was blocked with $5 \%$ goat serum in phosphate-buffered saline containing $0.2 \%$ Triton X-100 and $1 \%$ bovine serum albumin (Sigma-Aldrich) for $1 \mathrm{~h}$. After incubation with rabbit polyclonal antibodies against claudin-3 (1:400, Invitrogen) in $0.1 \%$ bovine serum albumin (BSA) overnight at $4{ }^{\circ} \mathrm{C}$, the sections were washed and incubated with $\mathrm{Cy} 3$-conjugated goat anti-rabbit IgG antibody (1:400, Invitrogen) for $1 \mathrm{~h}$ at $25^{\circ} \mathrm{C}$. Images were obtained with a fluorescence microscope (Nikon 80i, Sendai, Japan).

\subsection{Cell Culture}

The human CRC cell lines, HT-29 and DLD-1 cells, and normal HEK293 cell line were purchased from American Type Culture Collection (ATCC, Manassas, VA, USA). All cells were cultured in Dulbecco's modified eagle's medium (DMEM ) supplemented with $10 \%$ fetal bovine serum and $1 \%$ penicillin/streptomycin (Life Technologies) at $37^{\circ} \mathrm{C}$ in the presence of $5 \% \mathrm{CO}_{2}$.

The cells were treated with recombinant human SCF (rhSCF) (R\&D System, Minneapolis, MN, USA) in a gradient of concentrations $(0,25,50,100 \mathrm{ng} / \mathrm{mL})$ or for different time $(0,6,12,24,36,48 \mathrm{~h})$ or phorbol 12-O-tetradecanoate-13-acetate (TPA, $20 \mathrm{nM}$; Sigma-Aldrich) for different times (3, 6, 12, $24 \mathrm{~h})$. After serum starvation overnight, $\mathrm{rhSCF}(50 \mathrm{ng} / \mathrm{mL})$ was added into the medium $1 \mathrm{~h}$ after specific inhibitor treatment including SP600125 (JNK inhibitor, $10 \mu \mathrm{M}$; Sigma-Aldrich), SB203580 (p38 inhibitor, $10 \mu \mathrm{M}$; Sigma-Aldrich) and U0126 (extracellular signal regulated kinase (ERK) inhibitor, $10 \mu \mathrm{M}$; Sigma-Aldrich), respectively. The cells were harvested $36 \mathrm{~h}$ later and used for further experiments.

\subsection{Lentiviral Vector Construction and Infection}

To perform lentivirus mediated overexpression of c-kit, HT-29 cells were seeded in a 6-well plate and infected with the c-kit lentiviral vector (GV287, GeneChem, Shanghai, China) when 30\% confluency was reached. The infection efficiency was evaluated by the enhanced green fluorescent protein (EGFP) expression with an inverted florescence phase contrast microscope (Leica DMI3000 B, Brunswick, Germany).

\subsection{Construction of Plasmids}

The promoter region of the human $C L D N-3$ gene was amplified by PCR and then subcloned into Bgl II and Hind III sites of pGL3-basic plasmid (Promega, Madison, WI, USA) upstream of a luciferase reporter gene. The mutant AP-1 binding site was generated using a QuickChange Lightning Site-Directed Mutagenesis Kit (Agilent Technologies, Santa Clara, CA, USA). The primers used in construction or mutation are listed in Supplementary Table S2. AP-1 plasmid was purchased from ViGene (Rockville, MD, USA).

\subsection{Luciferase Reporter Assay}

HEK293T cells seeded in 96-well plates were transfected with CLDN-3 promoter mimics luciferase reporter plasmid, AP-1 vector, and Renilla luciferase expression vector (Promega) using Lipofectamin 2000 (Life Technologies). Luciferase activities were measured at $24 \mathrm{~h}$ after transfection using a Dual-Glo Luciferase Assay kit (Promega) and firefly luciferase activities were normalized to Renilla luciferase activities.

\subsection{Chromatin Immunoprecipitation (ChIP)}

HT-29 cells were exposed to DMSO (Sigma, USA), rhSCF, rhSCF plus SP600125, and SP600125, respectively and then treated with $1 \%$ formaldehyde to crosslink the protein to DNA. ChIP assay and real-time PCR were performed as previously described [34]. The primers are shown in Supplementary Table S3. 


\subsection{Animals}

Wads $^{-/+}$mice on a C57BL/ 6 background were purchased from the Model Animal Research Center of Nanjing University (Nanjing, China) and maintained in the standard environment conditions. Wild type (WT) and $\mathrm{Wads}^{-/-}$mice were obtained by mating Wads ${ }^{-/+}$parents as previously described [20]. Mice were genotyped by their distinct differences in fur pigmentation: black for WT, piebaldism for $\mathrm{Wads}^{-/+}$, and white for Wads ${ }^{-/-}$mice.

\subsection{Statistics}

Results were presented as the means \pm SEM and analyzed using a student's $t$-test or one-way ANOVA with the SPSS 23.0 software (IBM Corporation, New York, NY, USA). A $p$-value of 0.05 or less was considered statistically significant.

\section{Conclusions}

In conclusion, the present study clearly revealed that activation of SCF/c-kit/JNK/AP-1 signaling axis could obviously up-regulate claudin-3 expression in the colonic epithelium and colorectal carcinoma. Our results provide insight into the molecular mechanisms underlying the regulation of claudin-3 expression which will help us to develop specific drugs for the clinical treatment of high claudin-3 related diseases.

Supplementary Materials: Supplementary materials can be found at www.mdpi.com/1422-0067/18/4/765/s1.

Acknowledgments: This study was supported by the National Natural Science Foundation of China (31371220), the Beijing Natural Science Foundation $(5164029,5172008,7172021)$, the Scientific Research Key Program of Beijing Municipal Commission of Education (KM201610025002), Beijing Training Programme Foundation for Key Talents (2015000020124G114), and Open Project of Beijing Key Laboratory of Cancer Invasion and Metastasis Research (2015ZLQX06).

Author Contributions: Yaxi Wang, Tingyi Sun, and Dandan Li designed and performed the experiments; Shu Yang, Yaxi Wang, and Tingyi Sun analyzed the data; Haimei Sun contributed reagents/materials/analysis tools; Yaxi Wang also wrote a draft of the paper; and Deshan Zhou conceived the experiments and revised the paper.

Conflicts of Interest: The authors declare no conflict of interest.

\section{Abbreviations}

$\begin{array}{ll}\text { TJs } & \text { Tight junctions } \\ \text { CRC } & \text { Colorectal cancer } \\ \text { JNK } & \text { C-Jun N-terminal kinase } \\ \text { ChIP } & \text { Chromatin immunoprecipitation } \\ \text { AP-1 } & \text { Activator protein-1 } \\ \text { RTK } & \text { Receptor tyrosine kinases } \\ \text { EGFR } & \text { Epidermal growth factor receptor } \\ \text { MAPK } & \text { Mitogen-activated protein kinase } \\ \text { SCF } & \text { Stem cell factor } \\ \text { rhSCF } & \text { Recombinant human SCF } \\ \text { ERK } & \text { Extracellular signal-regulated kinases } \\ \text { IGF-1 } & \text { Insulin-like growth factor 1 } \\ \text { VEGFR } & \text { Vascular endothelial growth factor }\end{array}$

\section{References}

1. Singh, A.B.; Dhawan, P. Claudins and cancer: Fall of the soldiers entrusted to protect the gate and keep the barrier intact. Semin. Cell Dev. Biol. 2015, 42, 58-65. [CrossRef] [PubMed]

2. Cereijido, M.; Contreras, R.G.; Shoshani, L. Cell Adhesion, Polarity, and Epithelia in the Dawn of Metazoans. Physiol. Rev. 2004, 84, 1229-1262. [CrossRef] [PubMed]

3. Lal-Nag, M.; Morin, P.J. The claudins. Genome Biol. 2009, 10, 235. [CrossRef] [PubMed] 
4. Fernandez-Blanco, J.A.; Estevez, J.; Shea-Donohue, T.; Martinez, V.; Vergara, P. Changes in Epithelial Barrier Function in Response to Parasitic Infection: Implications for IBD Pathogenesis. J. Crohns Colitis 2015, 9, 463-476. [CrossRef] [PubMed]

5. Choi, Y.L.; Kim, J.; Kwon, M.J.; Choi, J.S.; Kim, T.J.; Bae, D.S.; Koh, S.S.; In, Y.H.; Park, Y.W.; Kim, S.H.; et al. Expression profile of tight junction protein claudin-3 and claudin-4 in ovarian serous adenocarcinoma with prognostic correlation. Histol. Histopathol 2007, 22, 1185-1195. [PubMed]

6. Cocco, E.; Casagrande, F.; Bellone, S.; Richter, C.E.; Bellone, M.; Todeschini, P.; Holmberg, J.C.; Fu, H.H.; Montagna, M.K.; Mor, G.; et al. Clostridium perfringens enterotoxin carboxy-terminal fragment is a novel tumor-homing peptide for human ovarian cancer. BMC Cancer 2010, 10, 349. [CrossRef] [PubMed]

7. Kominsky, S.L.; Vali, M.; Korz, D.; Gabig, T.G.; Weitzman, S.A.; Argani, P.; Sukumar, S. Clostridium perfringens enterotoxin elicits rapid and specific cytolysis of breast carcinoma cells mediated through tight junction proteins claudin-3 and -4. Am. J. Pathol. 2004, 164, 1627-1633. [CrossRef]

8. Long, H.; Crean, C.D.; Lee, W.H.; Cummings, O.W.; Gabig, T.G. Expression of Clostridium perfringens enterotoxin receptors claudin-3 and claudin-4 in prostate cancer epithelium. Cancer Res. 2001, 61, 7878-7881. [PubMed]

9. Montgomery, E.; Mamelak, A.J.; Gibson, M.; Maitra, A.; Sheikh, S.; Amr, S.S.; Yang, S.; Brock, M.; Forastiere, A.; Zhang, S.; et al. Overexpression of claudin proteins in esophageal adenocarcinoma and its precursor lesions. Appl. Immunohistochem. Mol. Morphol. 2006, 14, 24-30. [CrossRef] [PubMed]

10. Eftang, L.L.; Esbensen, Y.; Tannaes, T.M.; Blom, G.P.; Bukholm, I.R.; Bukholm, G. Up-regulation of CLDN1 in gastric cancer is correlated with reduced survival. BMC Cancer 2013, 13, 586. [CrossRef] [PubMed]

11. Kinugasa, T.; Huo, Q.; Higashi, D.; Shibaguchi, H.; Kuroki, M.; Tanaka, T.; Futami, K.; Yamashita, Y.; Hachimine, K.; Maekawa, S.; et al. Selective up-regulation of claudin-1 and claudin-2 in colorectal cancer. Anticancer Res. 2007, 27, 3729-3734. [CrossRef]

12. Tabaries, S.; Siegel, P.M. The role of claudins in cancer metastasis. Oncogene 2017, 36, 1176-1190. [CrossRef] [PubMed]

13. Dhawan, P.; Ahmad, R.; Chaturvedi, R.; Smith, J.J.; Midha, R.; Mittal, M.K.; Krishnan, M.; Chen, X.; Eschrich, S.; Yeatman, T.J.; et al. Claudin-2 expression increases tumorigenicity of colon cancer cells: Role of epidermal growth factor receptor activation. Oncogene 2011, 30, 3234-3247. [CrossRef] [PubMed]

14. Ikari, A.; Sato, T.; Watanabe, R.; Yamazaki, Y.; Sugatani, J. Increase in claudin-2 expression by an EGFR/MEK/ERK/c-Fos pathway in lung adenocarcinoma A549 cells. Biochim. Biophys. Acta 2012, 1823, 1110-1118. [CrossRef] [PubMed]

15. Fujii, N.; Matsuo, Y.; Matsunaga, T.; Endo, S.; Sakai, H.; Yamaguchi, M.; Yamazaki, Y.; Sugatani, J.; Ikari, A. Hypotonic Stress-induced Down-regulation of Claudin-1 and -2 Mediated by Dephosphorylation and Clathrin-dependent Endocytosis in Renal Tubular Epithelial Cells. J. Biol. Chem. 2016, 291, 24787-24799. [CrossRef] [PubMed]

16. Kim, B.; Breton, S. The MAPK/ERK-Signaling pathway regulates the expression and distribution of tight junction proteins in the mouse proximal epididymis. Biol. Reprod. 2016, 94, 22. [CrossRef] [PubMed]

17. Ikari, A.; Atomi, K.; Yamazaki, Y.; Sakai, H.; Hayashi, H.; Yamaguchi, M.; Sugatani, J. Hyperosmolarityinduced up-regulation of claudin-4 mediated by NADPH oxidase-dependent $\mathrm{H}_{2} \mathrm{O}_{2}$ production and Sp1/c-Jun cooperation. Biochim. Biophys. Acta 2013, 1833, 2617-2627. [CrossRef] [PubMed]

18. Bellone, G.; Smirne, C.; Carbone, A.; Buffolino, A.; Scirelli, T.; Prati, A.; Solerio, D.; Pirisi, M.; Valente, G.; Nano, M.; et al. KIT/stem cell factor expression in premalignant and malignant lesions of the colon mucosa in relationship to disease progression and outcomes. Int. J. Oncol. 2006, 29, 851-859. [CrossRef] [PubMed]

19. Chen, J.S.; Chen, K.T.; Fan, C.W.; Han, C.L.; Chen, Y.J.; Yu, J.S.; Chang, Y.S.; Chien, C.W.; Wu, C.P.; Hung, R.P.; et al. Comparison of membrane fraction proteomic profiles of normal and cancerous human colorectal tissues with gel-assisted digestion and iTRAQ labeling mass spectrometry. FEBS J. 2010, 277, 3028-3038. [CrossRef] [PubMed]

20. Tan, J.; Yang, S.; Shen, P.; Sun, H.; Xiao, J.; Wang, Y.; Wu, B.; Ji, F.; Yan, J.; Xue, H.; et al. C-kit signaling promotes proliferation and invasion of colorectal mucinous adenocarcinoma in a murine model. Oncotarget 2015, 6, 27037-27048. [CrossRef] [PubMed] 
21. De Souza, W.F.; Fortunato-Miranda, N.; Robbs, B.K.; de Araujo, W.M.; de-Freitas-Junior, J.C.; Bastos, L.G.; Viola, J.P.; Morgado-Diaz, J.A. Claudin-3 overexpression increases the malignant potential of colorectal cancer cells: Roles of ERK1/2 and PI3K-Akt as modulators of EGFR signaling. PLoS ONE 2013, 8, e74994. [CrossRef] [PubMed]

22. Ashida, R.; Tominaga, K.; Sasaki, E.; Watanabe, T.; Fujiwara, Y.; Oshitani, N.; Higuchi, K.; Mitsuyama, S.; Iwao, H.; Arakawa, T. AP-1 and colorectal cancer. Inflammopharmacology 2005, 13, 113-125. [CrossRef] [PubMed]

23. Rauhavirta, T.; Lindfors, K.; Koskinen, O.; Laurila, K.; Kurppa, K.; Saavalainen, P.; Maki, M.; Collin, P.; Kaukinen, K. Impaired epithelial integrity in the duodenal mucosa in early stages of celiac disease. Transl. Res. 2014, 164, 223-231. [CrossRef] [PubMed]

24. Komljenovic, D.; Sandhoff, R.; Teigler, A.; Heid, H.; Just, W.W.; Gorgas, K. Disruption of blood-testis barrier dynamics in ether-lipid-deficient mice. Cell Tissue Res. 2009, 337, 281-299. [CrossRef] [PubMed]

25. Morin, P.J. Claudin proteins in human cancer: Promising new targets for diagnosis and therapy. Cancer Res. 2005, 65, 9603-9606. [CrossRef] [PubMed]

26. De Oliveira, S.S.; de Oliveira, I.M.; de Souza, W.; Morgado-Diaz, J.A. Claudins upregulation in human colorectal cancer. FEBS Lett. 2005, 579, 6179-6185. [CrossRef] [PubMed]

27. Kwon, M.J.; Kim, S.S.; Choi, Y.L.; Jung, H.S.; Balch, C.; Kim, S.H.; Song, Y.S.; Marquez, V.E.; Nephew, K.P.; Shin, Y.K. Derepression of CLDN3 and CLDN4 during ovarian tumorigenesis is associated with loss of repressive histone modifications. Carcinogenesis 2010, 31, 974-983. [CrossRef] [PubMed]

28. Singh, A.B.; Harris, R.C. Epidermal growth factor receptor activation differentially regulates claudin expression and enhances transepithelial resistance in Madin-Darby canine kidney cells. J. Biol. Chem. 2004, 279, 3543-3552. [CrossRef] [PubMed]

29. Hatakeyama, N.; Kojima, T.; Iba, K.; Murata, M.; Thi, M.M.; Spray, D.C.; Osanai, M.; Chiba, H.; Ishiai, S.; Yamashita, T.; Sawada, N. IGF-I regulates tight-junction protein claudin-1 during differentiation of osteoblast-like MC3T3-E1 cells via a MAP-kinase pathway. Cell Tissue Res. 2008, 334, 243-254. [CrossRef] [PubMed]

30. Laakkonen, J.P.; Lappalainen, J.P.; Theelen, T.L.; Toivanen, P.I.; Nieminen, T.; Jauhiainen, S.; Kaikkonen, M.U.; Sluimer, J.C.; Yla-Herttuala, S. Differential regulation of angiogenic cellular processes and claudin-5 by histamine and VEGF via PI3K-signaling, transcription factor SNAI2 and interleukin-8. Angiogenesis 2017, 20, 109-124. [CrossRef] [PubMed]

31. Wisdom, R.; Johnson, R.S.; Moore, C. C-Jun regulates cell cycle progression and apoptosis by distinct mechanisms. EMBO J. 1999, 18, 188-197. [CrossRef] [PubMed]

32. Ikari, A.; Watanabe, R.; Sato, T.; Taga, S.; Shimobaba, S.; Yamaguchi, M.; Yamazaki, Y.; Endo, S.; Matsunaga, T.; Sugatani, J. Nuclear distribution of claudin-2 increases cell proliferation in human lung adenocarcinoma cells. Biochim. Biophys. Acta 2014, 1843, 2079-2088. [CrossRef] [PubMed]

33. Van Itallie, C.M.; Tietgens, A.J.; Anderson, J.M. Visualizing the dynamic coupling of claudin strands to the actin cytoskeleton through ZO-1. Mol. Biol. Cell 2017, 28, 524-534. [CrossRef] [PubMed]

34. Yang, S.; Wu, B.; Sun, H.; Ji, F.; Sun, T.; Zhao, Y.; Zhou, D. Interrupted E2F1-miR-34c-SCF negative feedback loop by hyper-methylation promotes colorectal cancer cell proliferation. Biosci. Rep. 2015, 36, e00293. [CrossRef] [PubMed]

(C) 2017 by the authors. Licensee MDPI, Basel, Switzerland. This article is an open access article distributed under the terms and conditions of the Creative Commons Attribution (CC BY) license (http://creativecommons.org/licenses/by/4.0/). 